\title{
FLORÍSTICA, ECOLOGIA E POTENCIAL PAISAGISTICO DE PALMEIRAS DO PARQUE ZOOBOTÂNICO DO MUSEU PARAENSE EMILIO GOELDI, BELÉM - PA
}

\author{
Laura Suéllen Lisboa Ferreira ${ }^{1}$, José Amir Lima de Sousa², \\ Mário Augusto Gonçalves Jardim³
}

\section{RESUMO}

As palmeiras estão amplamente distribuídas em todos os ecossistemas amazônicos e são utilizadas integralmente por comunidades na alimentação, na produção comercial, artesanal e nos centros urbano como insumo paisagístico em ambiente interno e externo. Este estudo teve como objetivo identificar as palmeiras com potencial paisagístico e seus respectivos requerimentos ecológicos. O levantamento florístico foi realizado em 5,4 ha do Parque Zoobotânico do Museu Paraense Emílio Goeldi em parcelas de 10 x 10 m registrando-se e identificando das espécies e posteriormente caracterizando quanto ao hábito de crescimento, o status ecológico e o potencial paisagístico. Foram registrados 32 gêneros e 50 espécies com Attalea sp. em maior número de espécies. O hábito arbóreo foi dominante para 42 espécies (84\%), a status ecológico mostrou que 36 espécies são nativas da Amazônia (72\%) e a alta luminosidade foi o padrão para 18 espécies (36\%). As palmeiras do Jardim Botânico do Museu Paraense Emílio Goeldi compõem uma amostra significativa da diversidade de palmeiras da Amazônia como elementos para a conservação ex situ e valor paisagístico, ambiental, social e econômico para região.

Palavras-chave: Arecaceae; Potencial paisagístico; Paisagismo urbano.

Recebido em 29.04.2014 e aceito em 08.05.2015

1 Bióloga. Mestre em Ciências Ambientais. Bolsista de Programa de Capacitação Institucional. Museu Paraense Emilio Goeldi. SPZ/Flora do Parque Zoobotânico, Belém-Pará: lauraferreira@museu-goeldi.br

2 Eng.Agrônomo. Mestre em Agronomia. Técnico do Museu Paraense Emílio Goeldi. SPZ/Flora do Parque Zoobotânico, Belém-Pará: amir@museu-goeldi.br

3 Pesquisador Titular. Museu Paraense Emilio Goeldi. Coordenação de Botânica, Belém-Pará:jardim@museu-goeldi.br 


\section{FLORISTIC, ECOLOGY AND POTENTIAL OF THE PALM TREES OF THE PARQUE ZOOBOTÂNICO DO MUSEU PARAENSE EMILIO GOELDI, BELÉM-PA}

\section{ABSTRACT}

The palms are widely distributed in all Amazonian ecosystems and communities are fully used by the food, the shopping, craft production and urban centers such as landscaped input in internal and external environment. This study aimed to identify the potential landscaped with palm trees and their ecological requirements. Was realized in 5.4 ha of the Parque Zoobotânico do Museu Paraense Emilio Goeldi with registration, identification, characterization, ecological status and potential landscape. of species occurrents. The results showed 32 genera and 50 species with Attalea showing the greatest number of species registered. The species arboreus were dominant with 42 species (84\%), 36 species $(72 \%)$ are native to the Amazon and high brightness 18 species (36\%). Palm trees make up a significant proportion of the Amazon diversity of palm trees as elements for the ex situ conservation and landscape, environmental, social and economic value for the region.

Keywords: Collection; Propagation; Cultivation; Urban landscaping.

\section{INTRODUÇÃO}

A região amazônica é conhecida no contexto global no que diz respeito a sua conservação e preservação. Por essa razão, muito se tem debatido sobre o desmatamento e seu impacto na conservação da biodiversidade, assim como a manutenção das áreas verdes. Nesse sentido, o Parque Zoobotânico do Museu Paraense Emílio Goeldi, no município de Belém-PA, fornece, há décadas, contribuições de grande relevância na manutenção e conservação de espécies da fauna e flora amazônicas.

Fundado em 06 de outubro de 1866 por Domingos Soares Ferreira Pena e consolidado por Emílio Goeldi no período de 1894 a 1907, o Museu Goeldi nasceu de idealistas que acreditavam no futuro da região amazônica e da necessidade de pesquisar os recursos naturais, organizar coleções científicas e exposições públicas e prestar serviços à comunidade em geral, ao mesmo tempo em que chama a atenção para a conservação das espécies e do meio ambiente (AMARAL et al., 1993) 
Arecaceae abrange em torno de 200 gêneros em 2000 espécies com distribuição pantropical e ocupando quase todos os habitats terrestres tropicais e sub-tropicais; portanto, são as plantas mais características das florestas tropicais (MARTINS; FILGUEIRAS, 2006; SOUZA; LORENZI, 2008). Segundo Jardim e Cunha (1998a) e Lorenzi et al. (2010) a flora amazônica concentra um expressivo número de palmeiras totalizando entre 200 a 250 espécies e constituindo a maior diversidade de palmeiras do Brasil, com 35 dos 42 gêneros e cerca de 150 das 193-208 espécies reconhecidas para o país. Constituem um dos grupos ecologicamente mais importantes, com praticamente todas as suas partes aproveitadas, desde a alimentação até o uso medicinal; são utilizadas por comunidades tradicionais, que geram rendas substanciais e ecologicamente sustentáveis e por isso são consideradas como uma das mais importantes fontes de recursos vegetais para o homem (MIRANDA et al. 2001; SOUZA; LORENZI, 2008).

Na região Amazônia ainda se sabe pouco sobre a diversidade e a distribuição das palmeiras, pois se estima que os inventários não atingiram a suficiência amostral para caracterizá-las (HENDERSON et al., 1995), enquanto Rocha e Silva (2005) e Silva (2008a) ressaltaram que a maioria dos estudos realizados na Amazônia sobre distribuição e estrutura de espécies florestais tem excluído as palmeiras de pequeno porte da flora regional. As palmeiras amazônicas são utilizadas na confecção de artesanatos, na cobertura do teto, na produção de palmito, na construção de casas e abrigos; na alimentação e na indústria de óleo, entre outros. Muitas espécies são procuradas comercialmente em virtude dos aspectos paisagísticos (folhagem, porte e inflorescências) para jardins, praças, ruas e ambientes internos. No parque do Museu Goeldi, as palmeiras estão distribuídas em toda a área útil e servem como amostras vivas da biodiversidade amazônica demonstrando mais um motivo para sua conservação.

Este estudo teve como objetivo identificar as palmeiras com potencial paisagístico e seus respectivos requerimentos ecológicos visando incentivar ações para a conservação e o paisagismo urbano.

\section{MATERIAIS E MÉTODOS}

O estudo foi realizado em 5,4 ha do Parque Zoobotânico do Museu Paraense Emílio Goeldi, localizado no centro urbano da capital Belém no Estado do Pará. O clima é do tipo Afi (quente e úmido) (AMARAL et al., 1993). As médias pluviométricas mensais são iguais ou superiores a $60 \mathrm{~mm}$ (DIB, 2003). A média anual da umidade relativa do ar varia de $80 \%$ a $90 \%$ no período de dezembro a junho e as médias anuais de temperatura de $26^{\circ} \mathrm{C}$ com 
mínima de $23^{\circ} \mathrm{C}$ e máxima de $31^{\circ} \mathrm{C}$ (NOVAES; LIMA, 1986). O solo é do tipo Latossolo Amarelo com textura arenosa e uma deficiência de fósforo e potássio (AMARAL et al., 1993). É um remanescente florestal com 33\% de vegetação composta por aproximadamente 500 espécies entre ervas, cipó, arbustos e árvores de grande porte, estão representadas por aproximadamente 5000 indivíduos (CAVALCANTE, 2006).

A análise da composição florística foi realizada de março a julho de 2013 e de acordo com a metodologia adotada por Jardim et al. (2007) onde foram demarcadas aleatoriamente 10 parcelas de $50 \mathrm{~m} \times 50 \mathrm{~m}$ correspondente a 2,5 ha ou seja $50 \%$ da área total do Parque, portanto, com suficiência amostral adequada. Em cada parcela e com auxílio de um parabotânico as espécies foram identificadas em nível de gênero e espécie. Para classificação das espécies foi adotado o sistema de Cronquist (1981) e Uhl e Dransfield (1987) e a revisão da nomenclatura taxonômica foi de acordo com Lista de plantas da flora do Brasil (2012) e o The Plant List (2014).

As características ecológicas incluíram o hábito de crescimento, o status ecológico e o indicativo paisagístico. A classificação quanto o hábito de crescimento foi de acordo com Miranda et al. (2001) em cinco tipos: Arbóreo (geralmente solitário), Arbustivo (geralmente em touceiras), Acaule (estipe subterrâneo), Trepador e Erva (palmeiras com menos de 1 metro de altura). O status ecológico foi caracterizado em palmeira nativa e exótica (LORENZI et al., 2010) e o indicativo paisagístico os ambientes externo e interno por meio do padrão de luminosidade adequado a cada espécie em Alta, Média e Baixa.

\section{RESULTADOS E DISCUSSÃO}

Foram registrados 32 gêneros e 50 espécies caracterizadas quanto ao hábito de crescimento, status ecológico e o indicativo paisagístico com base no nível de luminosidade exigida (Tabela 1).

Tabela 1. Espécies de Arecaceae ocorrentes no Parque Zoobotânico do Museu Paraense Emílio Goeldi, Belém, Pará, Brasil (HC-hábito de crescimento: Arb-arbóreo, Arbu-arbustivo, Trep-trepador; SE-status ecológico: Nat-nativa, Exo-exótica e IP- indicativo paisagístico: A-alta, M-média, B-baixa)

Table 1. Species of the Arecaceae occurring in the Parque Zoobotânico do Museu Paraense Emílio Goeldi, Belém, Pará, Brazil (HC-growth habit; Arb-arboreous, Arbu-arbustive, Trep-climbing; SE-status ecological: Nat-native, Exo-exotic and IP-landscape: A-high, Maverage, B-low)

\begin{tabular}{llll}
\hline \multicolumn{1}{c}{ Espécie } & HC & SE & IP \\
\hline Acrocomia aculeata (Jacq.) Lodd. ex Mart. & Arb & Nat & M \\
Aiphanes aculeata Willd. & Arb & Nat & A \\
\hline
\end{tabular}




\begin{tabular}{|c|c|c|c|}
\hline Areca triandra Roxb. ex. Buch. Ham & Arb & Exo & A \\
\hline Astrocaryum aculeatum G. Mey & Arb & Nat & $\mathrm{M}$ \\
\hline Astrocaryum murumuru Mart. var. murumuru & Arb & Nat & M \\
\hline Astrocaryum vulgare Mart. & Arb & Nat & M \\
\hline Attalea attaleoides (Barb. Rodr.) Wess. Boer & Arb & Nat & $A$ \\
\hline Attalea dahlgreniana (Bondar) Wess. Boer & Arb & Nat & $A$ \\
\hline Attalea maripa (Aubl.) Mart. & Arb & Nat & $A$ \\
\hline Attalea phalerata Mart. ex. Spreng. & Arb & Nat & $A$ \\
\hline Attalea speciosa Mart. ex Spreng. & Arb & Nat & A \\
\hline Bactris gasipaes Kunth & Arbu & Nat & M \\
\hline Bactris major Jacq. var. major & Arbu & Nat & M \\
\hline Bactris maraja Mart. var. maraja & Arbu & Nat & M \\
\hline Bentinckia nicobarica (Kurz) Becc. & Arb & Exo & $\mathrm{B}$ \\
\hline Carpentaria acuminata (H. Wendl. \& Drude) Becc. & Arb & Exo & $\mathrm{B}$ \\
\hline Caryota urens $\mathrm{L}$. & Arb & Exo & $\mathrm{B}$ \\
\hline Chelyocarpus chuco (Mart.) H. E. Moore & Arb & Nat & M \\
\hline Chelyocarpus ulei Dammer & Arb & Nat & M \\
\hline Cocos nucifera L. & Arb & Exo & A \\
\hline Copernicia prunifera (Mill.) H.E. Moore & Arb & Nat & A \\
\hline Cyrtostachys renda Blume & Arb & Exo & $\mathrm{M}$ \\
\hline Desmoncus polyacanthos Mart. & Trep & Nat & $\mathrm{B}$ \\
\hline Dypsis lutescens (H. Wendl.) Beentje \& J. Dransf. & Arb & Exo & $\mathrm{B}$ \\
\hline Dypsis madagascariensis (Becc.) Beentje \& J. Dransf. & Arb & Exo & $\mathrm{B}$ \\
\hline Elaeis oleifera (Kunth) Cortés & Arb & Nat & A \\
\hline Euterpe oleracea Mart. & Arbu & Nat & M \\
\hline Euterpe precatoria Mart. var. precatoria & Arbu & Nat & M \\
\hline Geonoma laxiflora Mart. & Arbu & Nat & $\mathrm{B}$ \\
\hline Geonoma maxima subsp. maxima (Poit.) Kunth & Arbu & Nat & $\mathrm{B}$ \\
\hline Iriartea deltoidea Ruiz \& Pav. & Arb & Nat & $\mathrm{B}$ \\
\hline Leopoldinia pulchra Mart. & Arb & Nat & A \\
\hline Livistona chinensis (Jacq.) R. Br. ex. Mart. & Arb & Exo & A \\
\hline Livistona rotundifolia (Lam.) Mart. & Arb & Exo & A \\
\hline Manicaria saccifera Gaertn. & Arb & Nat & M \\
\hline Mauritia flexuosa L. f. & Arb & Nat & A \\
\hline Mauritiella armata (Mart.) Burret & Arb & Nat & A \\
\hline Oenocarpus bacaba Mart. & Arb & Nat & M \\
\hline Oenocarpus bataua Mart. & Arb & Nat & M \\
\hline Oenocarpus mapora H. Karst. & Arb & Nat & M \\
\hline Oenocarpus minor Mart. & Arb & Nat & M \\
\hline Phoenix roebelenii O'Brien & Arb & Exo & $A$ \\
\hline Phytelephas macrocarpa Ruiz \& Pav. & Arb & Nat & A \\
\hline $\begin{array}{l}\text { Ptychosperma macarthurii (H.Wendl. ex. H. J. Veitch) H. Wendl. ex. Hook. } \\
\text { f. }\end{array}$ & Arb & Exo & $A$ \\
\hline Rhapis excelsa (Thunb.) A. Henry & Arb & Exo & A \\
\hline Roystonea regia (Kunth) O. F. Cook & Arb & Exo & A \\
\hline Socratea exorrhiza (Mart.) H. Wendl. & Arb & Nat & M \\
\hline Syagrus cocoides Mart. & Arb & Nat & $A$ \\
\hline Syagrus inajai (Spruce) Becc. & Arb & Nat & $A$ \\
\hline Syagrus sancona (kunth) H. Karst. & Arb & Nat & A \\
\hline
\end{tabular}


O gênero Attalea apresentou o maior número de espécies com $A$. attaleoides, $A$. dahlgreniana, A. maripa, A. phalerata e A. speciosa seguido por Oenocarpus com $O$. bacaba, O. bataua, O. mapora e O. minor. Três gêneros foram compostos por três espécies, quatro gêneros por duas espécies e vinte e quatro gêneros por uma única espécie (Figura 1). Algumas espécies de palmeiras cultivadas no Parque estão ilustradas na Figura 2.

Figura 1. Gêneros com o respectivo número de espécies no levantamento florístico no Parque Zoobotânico do Museu Paraense Emilio Goeldi, Belém, Pará, Brasil

Figure 1. Genera where the number of species in the floristic survey in the Parque Zoobotânico do Museu Paraense Emilio Goeldi, Belém, Pará

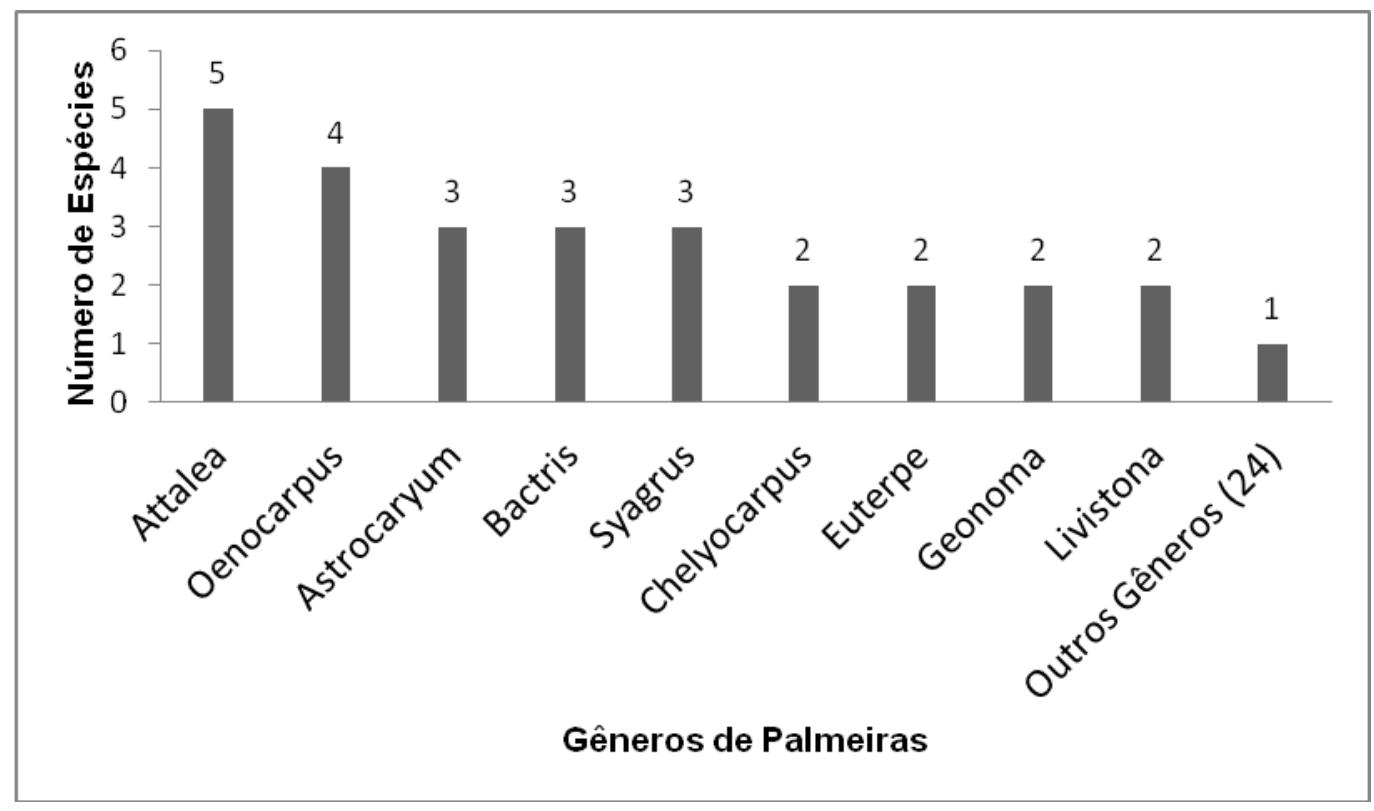

A quantidade de gêneros e espécies deste estudo está muito acima daqueles encontrados por Ribeiro et al. (2007) que registrou 11 gêneros e 18 espécies em 30,9 ha no Parque Municipal do Mindu em Manaus (AM); Miguel et al. (2007) com 6 gêneros e 6 espécies no Campus I da Universidade Grande Rio (RJ); Silva et al. (2007) com 12 gêneros e 12 espécies em parques urbanos de Recife (PE); Ribeiro e Azevedo (2009) com 8 gêneros e 8 espécies no Centro de Pesquisa da fauna e flora da Amazônia (CPPFAM) (AM); Silva e Ferraz (2012) com 3 gêneros e 3 espécies no Jardim Botânico de Recife (PE) e por Souza et al. (2013) que identificaram 6 gêneros e 7 espécies em 8,5 ha no Jardim Botânico Municipal Prefeito Carrol Meneghel, Americana (SP). Apenas Ribeiro et al. (2007) registraram Attalea e Oenocarpus com o maior número de espécies.

A comparação com esses estudos apenas ressalta que o banco de germoplasma de palmeiras do Parque Zoobotânico do Museu Emilio Goeldi tem significado como valor histórico, ambiental, biológico e genético. 
Figura 2. Espécies de palmeiras cultivadas no Parque Zoobotânico do Museu Paraense Emílio Goeldi: a. Aiphanes aculeata; b. Areca triandra; c. Oenocarpus minor; d. Bentinckia nicobarica; e. Mauritia flexuosa; f. Geonoma laxiflora; g. Iriartea deltoidea; h. Astrocaryum murumuru; i. Livistona chinensis; j. Socratea exorrizha; k. Ptychosperma macarthurii; I. Syagrus inajai.

Figure 2. Palm species cultivated in the Parque Zoobotânico do Museu Paraense Emílio Goeldi: a. Aiphanes aculeata; b. Areca triandra; c. Oenocarpus minor; d. Bentinckia nicobarica; e. Mauritia flexuosa; f. Geonoma laxiflora; g. Iriartea deltoidea; h. Astrocaryum murumuru; i. Livistona chinensis; j. Socratea exorrizha; k. Ptychosperma macarthurii; I. Syagrus inajai.

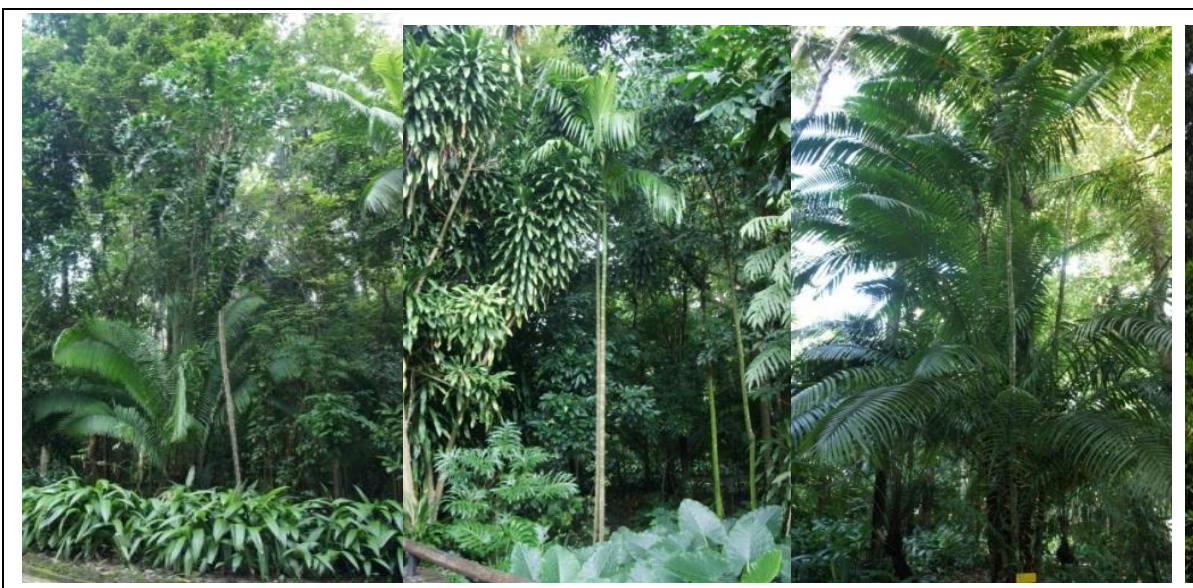

(a)

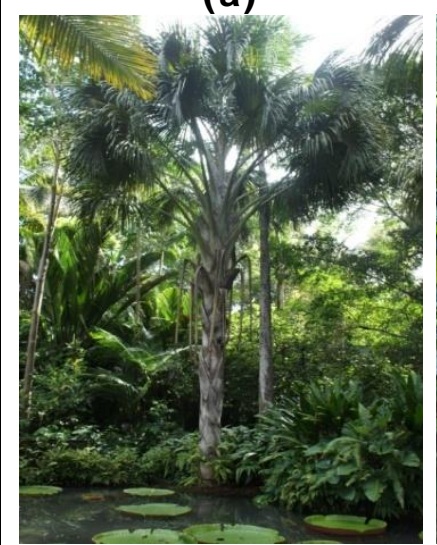

(e)

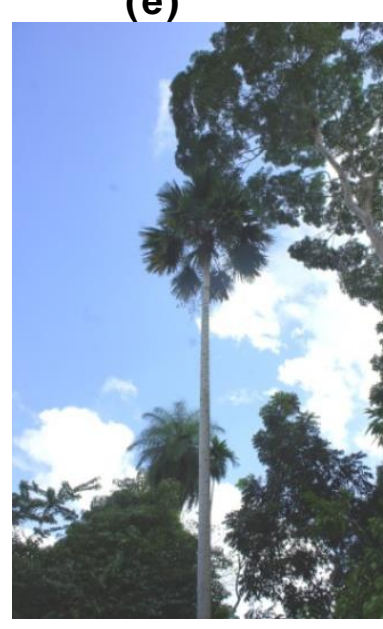

(i) (b)

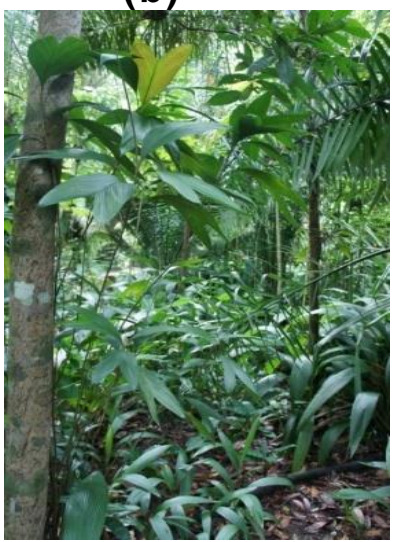

(f) (j)

(k)

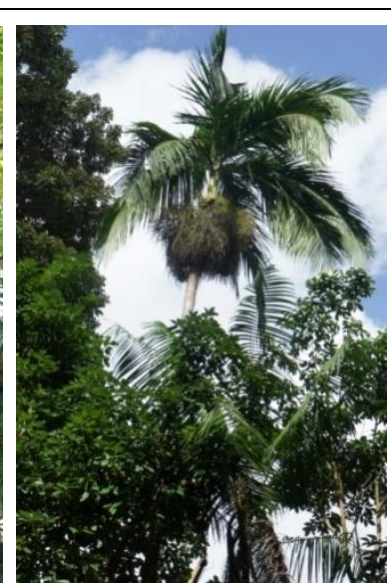

(d)

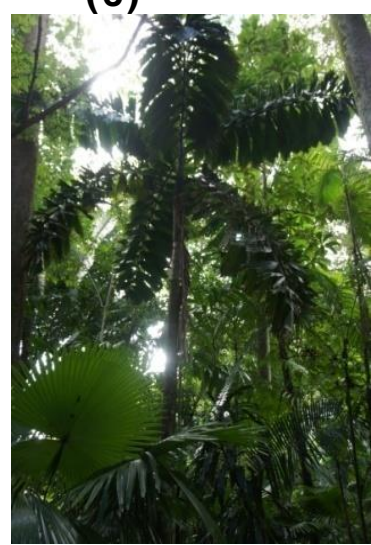

(g)

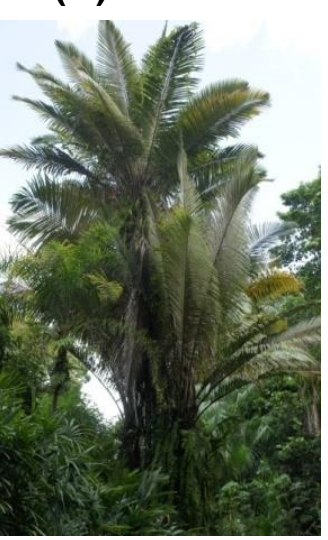

(h)

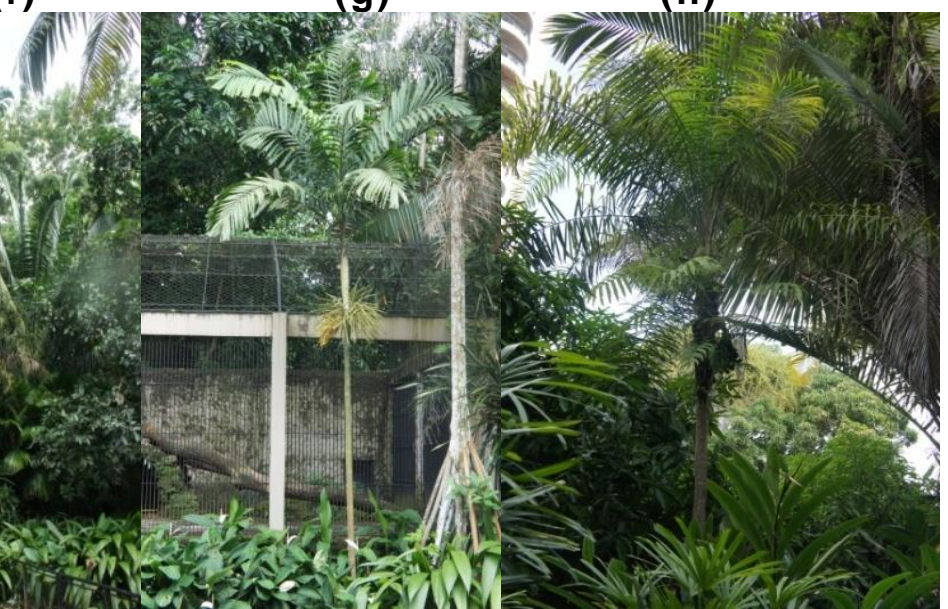

(I) 
O hábito arbóreo foi dominante com 42 espécies (84\%) seguido pelo arbustivo com 7 espécies (14\%) e trepador com 1 espécie (2\%). Quanto a origem, 36 espécies são nativas da região amazônica (72\%) e 14 espécies exóticas (28\%). Os estudos de Ribeiro et al. (2007), Miguel et al. (2007), Silva et al. (2007), Ribeiro e Azevedo (2009), Silva e Ferraz (2012) e Souza et al. (2013) mostraram que todas as palmeiras registradas tiveram o hábito arbóreo e há $85 \%$ nativas da região Norte, Nordeste e Sul do Brasil e 15\% de outros países.

O indicativo paisagístico com base na preferência das espécies por luminosidade mostrou que 23 espécies (46\%) preferem alta luminosidade, 18 espécies (36\%) luminosidade média e 9 espécies (18\%) baixa luminosidade. A preferência por alta luminosidade relacionada ao cultivo e adaptação em áreas abertas também foi registrada para as palmeiras por Ribeiro et al. (2007), Miguel et al. (2007), Silva et al. (2007), Ribeiro e Azevedo (2009), Silva e Ferraz (2012) e Souza et al. (2013). Este tipo de padrão estabelece que a maioria das palmeiras estejam adequadas ao plantio em áreas abertas ou ambientes externos e as demais aos ambientes com restrição a luminosidade ou ambientes paisagísticos internos.

Considerando o número de gêneros e espécies; hábito e procedência e a preferência por luminosidade, as palmeiras ocorrentes em 2,5 ha no Parque Zoobotânico do Museu Paraense Emilio Goeldi tiveram destaque no número de espécies em relação as outras áreas naturais e urbanas de conservação no Brasil. Este fato ressalta o valor conservacionista para este grupo plantas cujo potencial paisagístico é incontestável em qualquer parte do mundo.

\section{CONCLUSÕES}

As palmeiras do Jardim Botânico do Museu Paraense Emílio Goeldi compõem uma amostra significativa e quantitativa da diversidade de palmeiras nativas da Amazônia cujos elementos estruturais e adaptativos contribuem com indicativos para a conservação ex situ e valoração paisagística, ambiental, social e econômica para região.

\section{AGRADECIMENTOS}

Ao Conselho Nacional de Desenvolvimento Científico pelo apoio ao projeto de Bolsa de Produtividade "Palmeiras da Amazônia Oriental como indicadoras de conservação ambiental e qualidade de vida". CNPq-Processo 305667/2013-0. 


\section{REFERÊNCIAS}

AMARAL, I.G.; RUIVO, M.L.P.; DUTRA, F.C.; GAMA, J.R.V. Estudo preliminar sobre a fertilidade do solo no parque zoobotânico do Museu Paraense Emílio Goeldi. Bol. Mus.Para.Emílio Goeldi, Série Ciências da Terra, v.1., n.5, p.57-67, 1993.

CAVALCANTE, P.B. Guia botânico do Museu Goeldi. $3^{\underline{a}}$ ed. Belém: Museu Paraense Emílio Goeldi, 64p., 2006.

CRONQUIST, A. An integrated system of classification of flowering plants. New York: Columbia University Press, 248p.,1981.

DIB. Documento de Informações Básicas: Elaboração do Plano de Manejo do Parque Zoobotânico do Museu Paraense Emílio Goeldi. Belém: MPEG, 65 p., 2003.

HENDERSON, A.J.; GALEANO, G.; BERNAL, R. Field guide to the palms of the Americas. Princeton University Press, Princeton, USA. 352 pp., 1995.

JARDIM, M.A.G; CUNHA, A. C. Caracterização estrutural de populações nativas de palmeiras do estuário amazônico. Boletim do Museu Paraense Emílio Goeldi, Série Botânica, v.14, n.1, p.33-41, 1998a.

JARDIM, M. A. G.; SANTOS, G.C.S.; MEDEIROS, T.D.S.; FRANCEZ, D.C. Diversidade e estrutura de palmeiras em floresta de várzea do estuário amazônico. Amazônia: Ciência \& Desenvolvimento, v.2, n.4, p.7-24, 2007.

LISTA DE ESPÉCIES DA FLORA DO BRASIL, 2012. Jardim Botânico do Rio de Janeiro. www.floradoBrasil.jbrj.gov.br

LORENZI, H.; NOBLICK, L.R.; KAHN, F.; FERREIRA, E. Flora brasileira - Arecaceae (Palmeiras). Nova Odessa: Plantarum. 384 p.,2010.

MARTINS, R. C.; FILGUEIRAS, T. S. Arecaceae. In: CAVALCANTI, T. B. Flora do Distrito Federal, Brasil. Brasília: Embrapa Recursos Genéticos e Biotecnologia, p.47-82, 2006.

MIGUEL, J.R.; SILVA, M.A.M.; DUQUE,J.C.C. Levantamento de palmeiras (Arecaceae) cultivadas na Universidade do Grande Rio, Campus I - Duque de Caxias, Rio de Janeiro (Brasil). Saúde \& Ambiente, v.2, n.1, p.26-36, 2007.

MIRANDA, I. P. A.; RABElO, A.; BUENO, C. R.; BARBOSA, E. M.; RIBEIRO, M. N. S. Frutos de palmeiras da Amazônia. Manaus, Ministério de Ciência e Tecnologia, Instituto Nacional de Pesquisa da Amazônia. 120p., 2001. 
NOVAES, S.F.C.; LIMA, M.F.C. Aves da grande Belém. Municípios de Belém e Ananindeua. Belém: Museu Paraense Emílio Goeldi (coleção Emilia Snethlage), 1998. 415p. In: O Museu Paraense Emílio Goeldi. - São Paulo: Banco safra, p.32-36, 1986.

RIBEIRO, I.A.S.; ARAÚJO, M.G.P.; SANTANA, A.F. Palmeiras do Parque Municipal do Mindu, Manaus, Amazonas, Brasil. Revista Brasileira de Biociências, v.5, supl.,p.888-890, 2007.

RIBEIRO, I.A.S.; AZEVEDO, A.R. Catalogação das palmeiras (Arecaceae) do Zôo do Centro de Instrução de Guerra na Selva (CIGS) - Centro de Pesquisa da Fauna e Flora da Amazônia (CPPFAM) - Manaus, Amazonas - Brasil. Anais... da $61^{\circ}$ Reunião Anual da SBPC, Manaus (AM), p.51-52, 2009.

ROCHA, A. E. S.; SILVA, M. F. F. Aspectos fitossociológicos, florísticos e etnobotânicos das palmeiras (Arecaceae) de floresta secundária no município de Bragança, PA, Brasil. Acta Botanica Brasilica, v.19, n.3, p.657-667, 2005.

SILVA, L. S. A importância do babaçu (Atallea speciosa Mart. ex spreng.) no processo sucessional em áreas agrícolas no sudeste do Estado do Pará. 41p. Dissertação (Mestrado). Universidade Federal Rural da Amazônia e Museu Paraense Emílio Goeldi, Belém. 2008a.

SILVA, R.A.P.; FERRAZ, E.M.N. Espécies exóticas em um fragmento de Mata Atlântica: ameaças à biodiversidade local. Revista CIENTEC, v.4., n.2., p.56-64, 2012.

SILVA, L.R.; MEUNIER, I.M.J.; FREITAS, A.M.M. Riqueza e densidade de árvores, arvoretas e palmeiras em parques urbanos de Recife, Pernambuco, Brasil. REVSBAU, v.2, n.4, p.34-49, 2007.

SOUZA, V.C.; LORENZI, H. Botânica Sistemática. Guia ilustrado para identificação das famílias de fanerógamas nativas e exóticas no Brasil, baseado em APG II. Instituto Plantarum, Nova Odessa, SP. 704 p., 2008.

SOUZA, V.C.; NALON, C.F.; TONON, D. Árvores e palmeiras do Jardim Botânico Municipal Prefeito Carrol Meneghel, Americana (SP). REVSBAU, v.8, n.1, p.100-111, 2013.

THE PLAN LIST, 2014. Disponível em: <www.theplantlist.org>

UHL, N. W.; DRANSFIELD, J. Genera palmarum: a classification of palms based on the Work of Harold E.Moore Jr. Kansas: Allen Press: Lawrence, 610p., 1987. 